Egypt. J. of Appl. Sci., 36 (5-6) 2021

\title{
THE PREDICTION OF DISABILITY TO SCAPULAR TRAINING IN PATIENTS WITH SHOULDER IMPINGEMENT SYNDROME
}

\author{
Hager T.K. Mohamed *; Enas F. Youssef ** ; A.M.M. Gad *** \\ and Dina M.A. Al Hamaky ****
}

* Demonstrator of Physical Therapy for Musculoskeletal Disorder and its Surgery, Faculty of Physical Therapy, Cairo University.

** Professor and chairman of Physical Therapy for Musculoskeletal Disorders and its Surgery, Faculty of Physical Therapy, Cairo University.

*** Assistant Professor of Orthopedic Surgery and sports injuries, Faculty of Medicine, Cairo University.

**** Lecturer of Physical Therapy for Musculoskeletal Disorders and its Surgery, Faculty of Physical Therapy, Cairo University.

Key Words: scapular training, shoulder impingement syndrome, clinical prediction rule, SPADI

\section{ABSTRACT}

Study design: prospective clinical trial study

Background: People with shoulder impingement syndrome (SIS) present alterations in the scapular kinematics 'scapular dyskinesis' when compared with asymptomatic individuals. Those patients consistently report disability, especially during overhead activities, which might hinder activities of daily living and for some sports movements. Objectives: To investigate if the disability can predict patient's response to scapular training. Methods: Forty-five patients between 18-45 years old, their mean age $(32.56 \pm 2.85)$ years and their mean body mass index (BMI) $(29 \pm 6) \mathrm{kg} / \mathrm{m}^{2}$, suffering from subacromial impingement syndrome assessed by Shoulder Pain and Disability Index (SPADI) before and after exercise program of scapular muscle training (Cools exercises), serratus anterior strength (supine punch) and scapular stabilization exercises for one month. Results: The Area under curve (AUC) showed excellent results with disability percent 0.94 (sensitivity 0.956 , specificity 0.156 ). Conclusion: disability is a predictor of treatment success in patients suffering from subacromial impingement syndrome treated with scapular training.

\section{INTRODUCTION}

Shoulder impingement syndrome (SIS) is a common complaint for all aged patients with different activity levels (Moezy et al., 2014). It is defined as compression and mechanical abrasion of the rotator cuff structures as they pass under the coracoacromial arch during arm elevation(Koester et al., 2005). People who are frequently doing 
repetitive overhead motions related to their professions or athletic activities are also at risk of SIS (Koester et al., 2005; Brumitt et al., 2006). Thus, their physical independence and jobs are in danger of being loosed, that have important socioeconomic implications (Moezy et al., 2014).

Optimal scapular positioning and scapular control during shoulder movements may be important for normal shoulder function (Ludewig and Reynolds, 2009). Alteration in scapular position and movement are important risk factors for subacromial impingement syndrome (Kibler et al., 2013Clarsen et al., 2014). Stability of the scapula depends on the surrounding musculature, so, altered muscle activity in the scapular muscles and loss of scapular stabilization is commonly believed to be a crucial factor contributing to shoulder impingement syndrome (Ludewig and Cook, 2000; Cools et al., 2003).

Most of the exercise protocols emphasise the importance of scapular training as an essential component of shoulder rehabilitation (Başkurt et al., 2011; struyf et al., 2013; Moezy et al., 2014; Shah et al., 2014; Aytar et al., 2015).

Clinical prediction rules (CPRs) are tools planned to improve decision making in clinical practice, based on a subset of predictor variables from the history and physical examination(Childs \& Cleland, 2006). Up to authors knowledge no study has recognized specific clinical examination variables that are predictive of which patients with the condition will respond successfully to scapular training.

\section{METHODS}

This study designed as a single arm clinical trial study that was conducted at outpatient clinic at faculty of physical therapy, Cairo University, between September 2020 and April 2021 months.

Forty-five patients (male and female) between the age 18 and 45 years old, referred by orthopaedic surgeon as subacromial impingement syndrome (stage I and II Neer's classification). The first examiner checked patient's eligibility to the study according to the inclusion and exclusion criteria:

Inclusion criteria: (NEER and Welsh RP., 1977; Lukasiewicz et al., 1999; Michener et al., 2009; Cook et al., 2013; Consigliere et al., 2018)

1. Age 18-45 years old patients diagnosed as subacromial impingement syndrome.

Patients included in this study as if they have at least 3 of the following 6 criteria:

- Positive "Neer sign": The examiner passively flexes the humerus with medial rotation to end-range with over pressure. The 
patient's facial expression and the reproduction of the pain confirm the presence of impingement.

- Positive "Hawkins sign": The shoulder is passively placed in approximately 90 degrees of flexion and is passively internally rotated to end-range with overpressure, reproducing the patient's pain.

- Pain with active shoulder elevation in the scapular plane.

- Pain with palpation of the rotator cuff tendons.

- Pain with resisted isometric abduction.

- A history of pain in the superior part of lateral arm.

2. Demonstration of a painful arc of the arm from 60 to 120 of flexion.

Exclusion criteria: (Saito et al., 2018)

1. Diagnosis of internal shoulder impingement.

2. A history of traumatic onset of shoulder pain.

3. Recent trauma of shoulder.

4. Torn tendons.

5. Ligamentous laxity based on a positive Sulcus and apprehension tests.

6. Numbness or tingling in the upper extremity.

7. Cervical discogenic problems.

8. Previous shoulder or cervical spine surgery.

9. Systemic illness.

10. Corticosteroid injection on the shoulder within 1 year of the study.

11. Evidence of central nervous system involvement, or the inability to comply with treatment.

All patients received explanation of the study objectives and procedures. If the patients fit in the study criteria, they asked to sign the written consent form to participate in the study. Also, they informed that the data collected would be submitted for publication.

\section{Instrumentations and procedures:}

Shoulder Pain and Disability Index (SPADI):

It is a self-report questionnaire established to measure the pain and disability associated with shoulder pathology in people with shoulder pain of musculoskeletal, neurogenic, or undetermined origin(Roy et al., 2009).

Test-retest reliability of the SPADI ranges from 0.84 to 0.91 , and the minimal clinically important difference ranges from 8 to 13.2 points (Roy et al., 2009).

The translated version of SPADI in the Arabic language showed excellent internal consistency and test-retest reliability and construct 
validity based on substantial correlations of Arabic SPADI with other methods of evaluation (Yahia et al., 2011). The SPADI is recommended for the evaluation of patients with shoulder dysfunction (Alsanawi et al., 2015).

\section{Pretest evaluation}

Shoulder function was assessed using the Arabic version of Shoulder Pain and Disability Index (SPADI).

Disability domain of SPADI (physical function, 8 items): each item scored on a visual analogue scale ranging from 0 to 10 , where 0 no difficulty and 10 so difficult require help (Roy et al., 2009).

Total disability score: ..... / $80 \times 100=\%$

Total percentage score ranging from 0 to 100 , where 0 best and 100 worst (Roy et al., 2009)

\section{a) Posttest evaluation}

Final evaluation was assessed after twelve sessions of scapular training by the second examiner.

Progression of:

\section{Interventions procedures}

- Shoulder disability by SPADI.

patients scheduled to join physical therapy three sessions per week for one month (Saito et al., 2018). Three sets of 10 repetitions for each exercise were given per session, with a 1-minute rest between sets (De Mey et al., 2012). Resistance was established by 10 repetition maximum (RM) testing, these interventions was achieved by the first examiner (Cools et al., 2007).

\section{Scapular muscle training (Cools exercises):}

These exercises are successful in restoration of upper trapezius, middle trapezius and lower trapezius muscle imbalances (Cools et al., 2007).

a. Forward flexion in side-lying, patient was in side-lying, shoulder in neutral position; patient performed forward sidelying position flexion in a horizontal plane to $135^{\circ}$.

b. Side-lying external rotation, patient was in side-lying with the shoulder in neutral position and the elbow flexed $90^{\circ}$; patient performed external rotation of the shoulder (with towel between trunk and elbow to avoid compensatory movements).

c. Horizontal abduction with external rotation, patient was in prone with the shoulders resting in $90^{\circ}$ forward flexion; patient performed horizontal abduction to horizontal position, with an 
additional external rotation of the shoulder at the end of the movement.

d. Prone extension, patient was in prone with the shoulders resting in $90^{\circ}$ forward flexion; patient performed extension to neutral position with the shoulder in neutral rotational position.

\section{b. Serratus anterior strength (supine punch):}

Supine (serratus anterior) punch done by lying supine on a bench, while keeping the shoulder at $90^{\circ}$ of flexion with the elbow extended maximally, then protracting the scapula to its ending rang (Escalante et al., 2020).

\section{Scapular stabilization exercises:}

Scapular-clock exercise enhanced the scapular motions of elevation, depression, protraction, and retraction also, enhance joint kinaesthesia and range of motion. The participant stood at the side of a plinth while putting his hand on a ball and move it to show 3, 6, 9 or 12 o'clock based on an imaginary clock he had on his mind. This exercise can performed by pressing a ball and replacing it on a wall (Moezy et al., 2014).

\section{Data Analysis:}

forty-five patients between the age 18 and 45 years old ( 41 female and 4 male), referred by orthopaedic surgeon as subacromial impingement syndrome (stage I and II Neer's classification).

First the patients were interviewed, and if they matched with the inclusion criteria, they were asked to join the study. Then the detailed history was recorded from the patient included (name, age, sex, occupation, duration of symptoms, and affected side).

\section{RESULTS:}

The results of the demographic data showed that their mean age was $(32.56 \pm 2.85)$ years and their mean body mass index was (BMI) $(29 \pm 6) \mathrm{kg} / \mathrm{m}^{2}$.

Table (1): Distribution of disability in percent among the participants and correlation between results of participants before and after using of the test, as regarding to.

\begin{tabular}{|c|l|l|l|}
\hline Variables & Mean \pm SD & Range (Min-Max) & P value \\
\hline Disability in percent & & & $\mathbb{q 0 . 0 0 1}$ \\
\hline$>$ Pre & $\mathbf{1 7} \pm 10$ & $\mathbf{4 3}(1-44)$ & $\mathbf{F 0 . 0 0 0 1}$ \\
\hline$>$ Post & $\mathbf{5 2} \pm 20$ & $\mathbf{7 4 ( 1 7 - 9 2 )}$ & $\mathbf{\$ 0 . 0 0 1}$ \\
\hline
\end{tabular}

T Test of Correlation (Pearson's correlation)

F T - test

+ One way anova test

There was highly statistically significant difference between results of participants pre and post as regarding to disability in percent. 


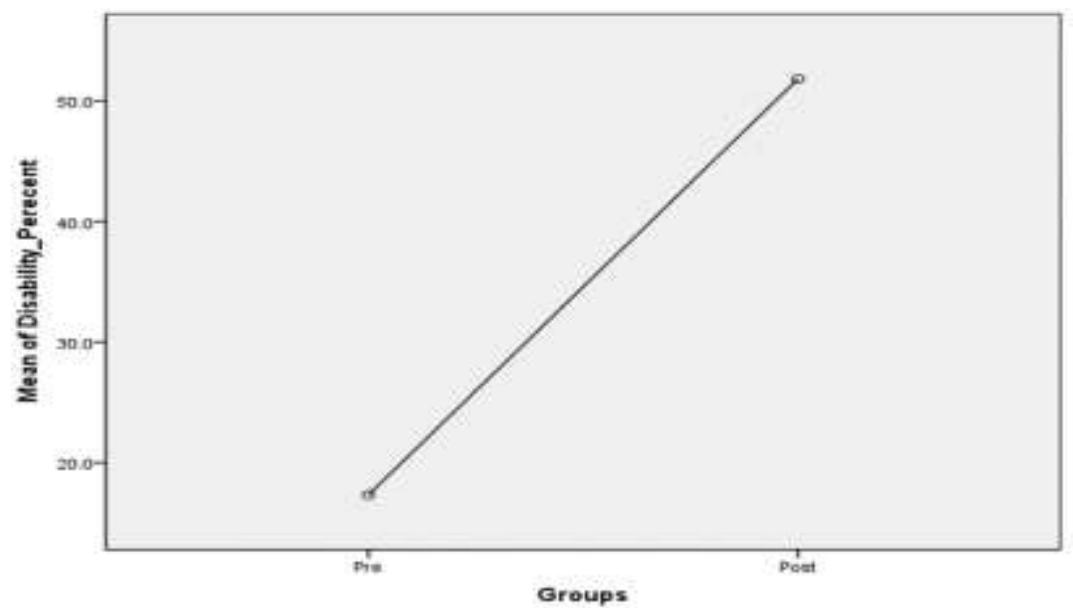

Figure 1: mean of pre and post disability in percent

Table (2): Description of the ROC curve, demonstrating AUC, Reliability, validity of interfering with this procedure, and Cut off points.

\begin{tabular}{|c|c|c|c|c|c|}
\hline \multicolumn{5}{|c|}{ Cut Off Points } \\
\hline & AUC & Result & $\begin{array}{c}\text { Positive if Greater } \\
\text { Than or Equal To }\end{array}$ & Sensitivity & $\begin{array}{c}1 \text { - } \\
\text { Specificity }\end{array}$ \\
\hline $\begin{array}{c}\text { Disability } \\
\text { in } \\
\text { percent }\end{array}$ & 0.94 & Excellent & 30.850 & 0.956 & 0.156 \\
\hline
\end{tabular}

AUC = Area under the curve

This table shows that disability Percent:

- Its sensitivity is 0.956

- Its 1 -specificity is 0.156

- Positive if greater than or equal To ${ }^{\mathrm{a}}$ is 30.85

- Area under carve is 0.94 ad result is excellent

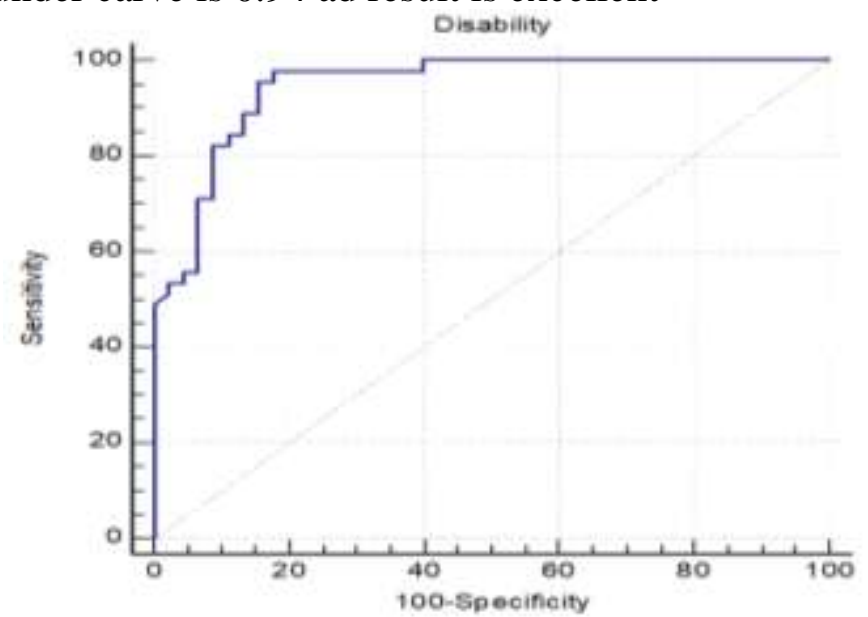

Figure 2: ROC curve, demonstrating AUC, Reliability, validity of interfering with this procedure, and Cut off points 


\section{DISCUSSION}

This study was conducted to investigate if disability can predict patient's response to scapular training in patients with subacromial impingement syndrome.

The results of the present study showed a significant difference regarding disability in percent pre and post the scapular training test $17 \pm 10$; $52 \pm 20$ respectively) ( $\mathrm{P}$ value $<0.05$ )

This study indicated that, scapular training is effective in improving function in patients suffering from subacromial impingement syndrome. These findings were similar to findings of (Bury et al., 2016; Reijneveld et al., 2017; Saito et al., 2018), who found that scapular training were effective in controlling pain, improving disability status and improving the scapular kinematics in patient with subacromial impingement syndrome.

Other study evaluated the effects of scapular stabilisation exercises immediately after surgery in patients with subacromial impingement syndrome, they found reduced pain and improved function after treatment(Park et al., 2013).

Furthermore, in multiple case series studies, positive effects on function and clinical measures of scapular positioning were found after a scapular exercise programme(De Mey et al., 2012; Worsley et al., 2013).

Our results showed that disability percent has an excellent sensitivity and is a positive predictor of the use of scapular training in patients with subacromial impingement syndrome if its value is greater than or equal $\mathrm{To}^{\mathrm{a}}$ is 30.85 .

Such findings come to an agreement with (Hotta et al., 2018) study analyzing the effect of scapular training protocol in impingement syndrome indicated that exercises focused on scapula are beneficial in decreasing impingement syndrome, there have been significant improvement in shoulder pain and disability index $(\mathrm{P}<0.01)$.

\section{CONCLUSION:}

Disability was a predictor of treatment success in patients suffering from subacromial impingement syndrome treated with scapular training.

\section{RECOMMENDATION:}

Future studies are recommended to investigate the effect of other factors on patient's outcome following scapular training.

\section{REFERENCES}

Alsanawi, H. A. ; A.Alghadir ; S. Anwer ; K.E.Roach and A.Alawaji (2015). Cross-cultural adaptation and psychometric properties of an Arabic version of the Shoulder Pain and Disability Index. International Journal of Rehabilitation Research, 38(3): 270-275.

Aytar, A. ; G.Baltaci ; T. Uhl ; H. Tuzun ; P. Oztop and M. Karatas (2015). The effects of scapular mobilization in patients with subacromial impingement syndrome: A randomized, double- 
blind, placebo-controlled clinical trial. Journal of Sport Rehabilitation, 24(2): 116-129.

Başkurt, Z. ; F. Başkurt ; N. Gelecek and M.H. Özkan (2011). The effectiveness of scapular stabilization exercise in the patients with subacromial impingement syndrome. Journal of Back and Musculoskeletal Rehabilitation, 24(3): 173-179.

Brumitt, J. ; J. Brumitt ; C. Willamette and F. Hospital (2006). Prescription Scapular-Stabilization Exercises: EarlyIntervention Prescription, 11(5): 15-18.

Bury, J. ; M.West ; G. Chamorro-Moriana and C. Littlewood (2016). Effectiveness of scapula-focused approaches in patients with rotator cuff related shoulder pain: A systematic review and meta-analysis. Manual Therapy, 25: 35-42.

Childs, J.D. and J.A.Cleland (2006). Development and application of clinical prediction rules to improve decision making in physical therapist practice. Physical Therapy, 86(1): 122-131.

Clarsen, B. ; R.Bahr ; S.H. Andersson ; R. Munk and G. Myklebust (2014). Reduced glenohumeral rotation, external rotation weakness and scapular dyskinesis are risk factors for shoulder injuries among elite male handball players: A prospective cohort study. British Journal of Sports Medicine, 48(17): 1327-1333.

Consigliere, P. ; O. Haddo ; O. Levy and G. Sforza (2018). Subacromial impingement syndrome: Management challenges. Orthopedic Research and Reviews, 10: 83-91.

Cook, C. ; K. Learman ; S. Houghton ; C. Showalter and B. O'Halloran (2013). The addition of cervical unilateral posterior-anterior mobilisation in the treatment of patients with shoulder impingement syndrome: A randomised clinical trial. Manual Therapy, 19(1): 18-24.

Cools, A.M. ; E.E.Witvrouw ; G.A. Declercq ; L.A. Danneels and D.C. Cambier (2003). Scapular muscle recruitment patterns: Trapezius muscle latency with and without impingement symptoms. American Journal of Sports Medicine, 31(4): 542-549.

Cools, A.M. ; V. Dewitte ; F. Lanszweert ; D. Notebaert ; A. Roets ; B. Soetens ; B. Cagnie and E.E. Witvrouw (2007). Rehabilitation of scapular muscle balance: Which exercises to prescribe? American Journal of Sports Medicine, 35(10): 1744-1751.

De Mey, K. ; L. Danneels ; B. Cagnie and A.M. Cools (2012). Scapular muscle rehabilitation exercises in overhead athletes with impingement symptoms: Effect of a 6-week training program on muscle recruitment and functional outcome. American Journal of Sports Medicine, 40(8): 1906-1915.

Escalante, G. ; D. Fine ; K. Ashworth and M.J. Kolber (2020). 
Progressive Exercise Strategies to Mitigate Shoulder Injuries Among Weight-Training Participants. Strength and Conditioning Journal, March, 1.

Hotta, G.H. ; A.L. Santos ; K.J. McQuade and A.S. de Oliveira (2018). Scapular-focused exercise treatment protocol for shoulder impingement symptoms: Three-dimensional scapular kinematics analysis. Clinical Biomechanics, 51, 76-81.

Kibler, W. Ben ; P.M. Ludewig ; P.W. Mcclure ; L.A. Michener ; K. Bak and A.D. Sciascia (2013). Clinical implications of scapular dyskinesis in shoulder injury: the 2013 consensus statement from the 'scapular summit .' 877-885.

Koester, M.C. ; M.S. George and J.E. Kuhn (2005). Shoulder impingement syndrome. American Journal of Medicine, 118(5): $452-455$.

Ludewig, P.M. and T.M.Cook (2000). Alterations in Shoulder Kinematics and Associated Muscle Activity in People With Symptoms of Shoulder Impingement. Physical Therapy, 80(3): 276-291.

Ludewig, P. M. and J. F.Reynolds (2009). The association of scapular kinematics and glenohumeral joint pathologies. Journal of Orthopaedic and Sports Physical Therapy, 39(2): 90-104.

Lukasiewicz, A.C. ; P. McClure ; L. Michener ; N. Pratt and B. Sennett (1999). Comparison of 3-dimensional scapular position and orientation between subjects with and without shoulder impingement. Journal of Orthopaedic and Sports Physical Therapy, 29(10): 574-586.

Michener, L.A. ; M.K. Walsworth ; W.C. Doukas and K.P. Murphy (2009). Reliability and Diagnostic Accuracy of 5 Physical Examination Tests and Combination of Tests for Subacromial Impingement.Archives of Physical Medicine and Rehabilitation, 90(11): 1898-1903.

Moezy, A. ; S. Sepehrifar and M.S. Dodaran (2014). The effects of scapular stabilization based exercise therapy on pain, posture, flexibility and shoulder mobility in patients with shoulder impingement syndrome: A controlled randomized clinical trial. Medical Journal of the Islamic Republic of Iran, 28(1):78.

NEER, C.S. and RP.Welsh (1977). the shouldr in sport. Orthop Clin North Am, 8: 583-591.

Park, S.I. ; Y.I. Cho ; J. Lee and K.I.M.Young-MIn (2013). Effects of shoulder stabilization exercise on pain and function recovery of shoulder impingement syndrome Patients. Journal of Physical Therapy Science, 25(12): 1359-1362.

Reijneveld, E.A.E. ; S. Noten ; L.A. Michener ; A. Cools and F. Struyf (2017). Clinical outcomes of a scapular-focused treatment in 
patients with subacromial pain syndrome: A systematic review. British Journal of Sports Medicine, 51(5): 436-441.

Roy, J.S. ; J.C. Macdermid, and L.J. Woodhouse (2009). Measuring shoulder function: A systematic review of four questionnaires. Arthritis Care and Research, 61(5): 623-632.

Saito, H. ; M.E. Harrold ; V. Cavalheri and L. McKenna (2018). Scapular focused interventions to improve shoulder pain and function in adults with subacromial pain: A systematic review and meta-analysis. Physiotherapy Theory and Practice, 34(9): 653-670.

Shah, M. ; J. Sutaria, and A. Khant (2014). Effectiveness of scapular stability exercises in the patient with the shoulder impingement syndrome Maulik. Indian Journal of Physical Therapy, 2(1): 79-84.

Worsley, P. ; M. Warner ; S. Mottram ; S. Gadola ; H.E.J.Veeger ; H. Hermens ; D. Morrissey ; P. Little ; C. Cooper ; A. Carr and M. Stokes (2013). Motor control retraining exercises for shoulder impingement: Effects on function, muscle activation, and biomechanics in young adults. Journal of Shoulder and Elbow Surgery, 22(4): e11-e19.

Yahia, A. ; M. Guermazi ; M.Khmekhem ; S. Ghroubi ; K. Ayedi and M.H. Elleuch (2011). Traduction en arabe et validation de l'indice ASES dans l'évaluation de l'incapacité fonctionnelle des pathologies de l'épaule. Annals of Physical and Rehabilitation Medicine, 54(2): 59-72.

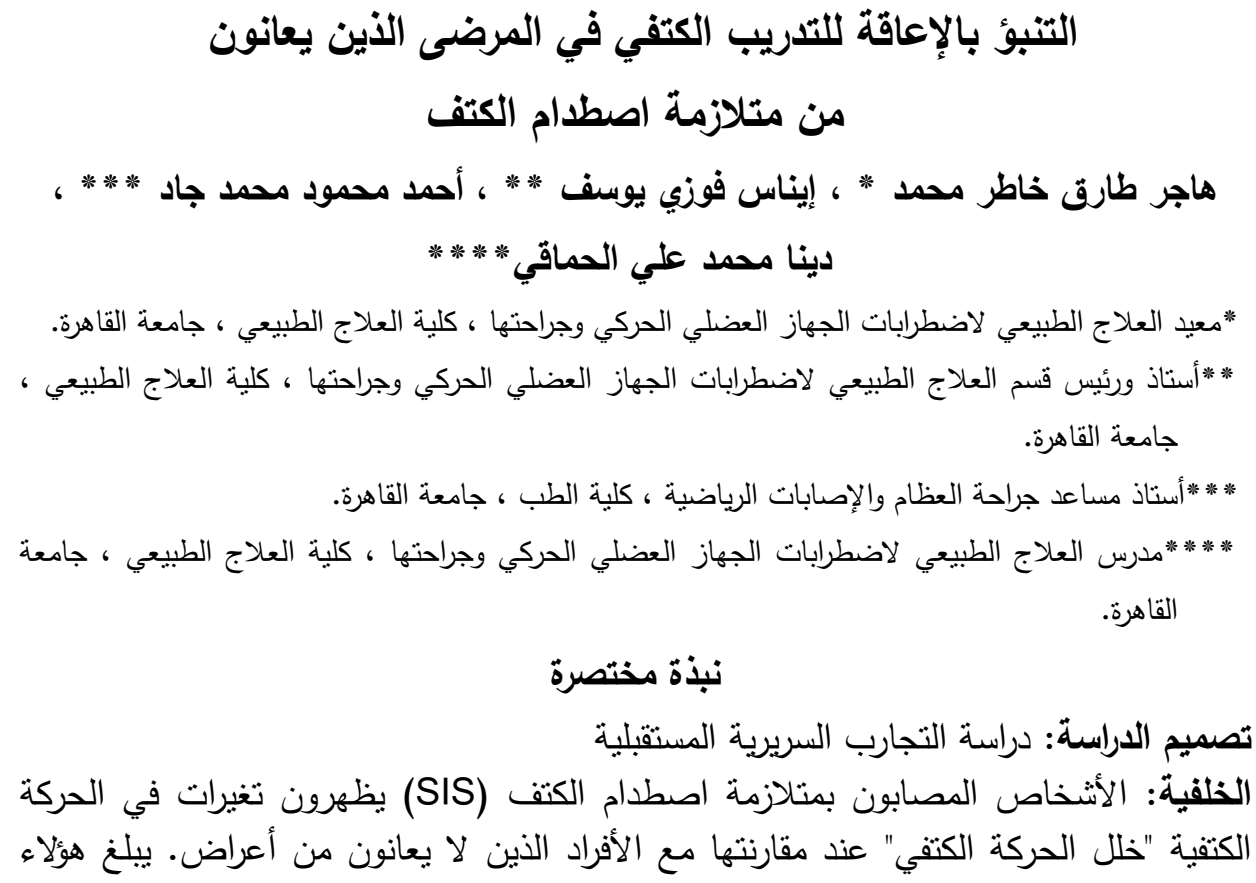




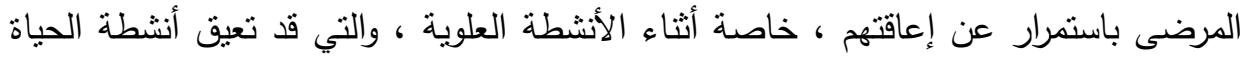

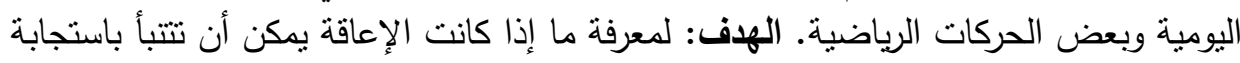

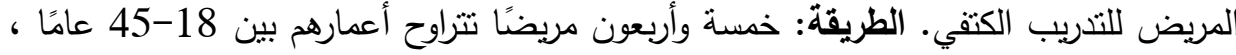

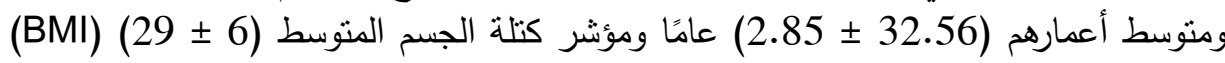

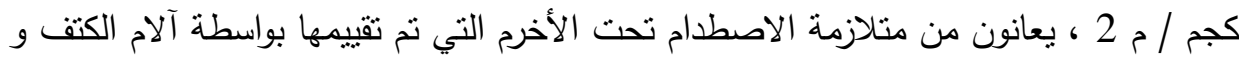

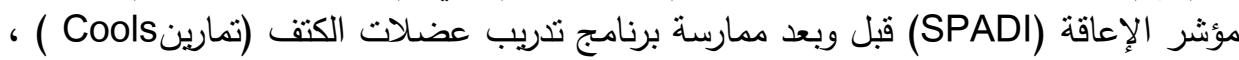
وتمارين القوة الأمامية المسننة (لكمة الاستلقاء) وتمارين التثبيت الكتفي لمدة شئنة شهر واحد. النتائج: أظهرت المنطقة تحت المنحنى (AUC) نتائج ممتازة مع نسبة إعارية العاقة 0.94 (حساسية

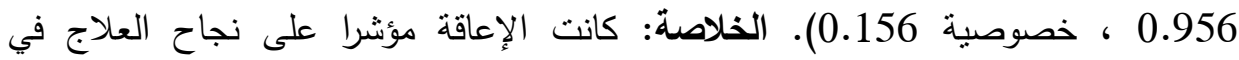
المرضى الذين يعانون من متلازمة الاصطدام تحت الأخرمي الذين عولجوا بتدريب كتفي.

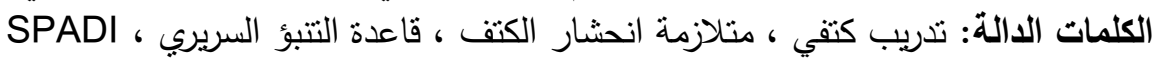

